\title{
Kernos
}

Revue internationale et pluridisciplinaire de religion grecque antique

2 | 1989

Varia

\section{Folklore et superstition en Grèce classique : Lamia torturée?}

\section{Monique Halm-Tisserant}

\section{(2) OpenEdition \\ Journals}

Édition électronique

URL : http://journals.openedition.org/kernos/236

DOI : 10.4000/kernos.236

ISSN : 2034-7871

\section{Éditeur}

Centre international d'étude de la religion grecque antique

\section{Édition imprimée}

Date de publication : 1 janvier 1989

Pagination : 67-82

ISSN : 0776-3824

\section{Référence électronique}

Monique Halm-Tisserant, « Folklore et superstition en Grèce classique : Lamia torturée ? », Kernos [En ligne], 2 | 1989, mis en ligne le 02 mars 2011, consulté le 10 décembre 2020. URL : http:// journals.openedition.org/kernos/236 ; DOI : https://doi.org/10.4000/kernos.236 
Kernos, 2 (1989), p. 67-82.

\section{FOLKLORE ET SUPERSTITION EN GRÈCE CLASSIQUE : LAMIA TORTURÉE ?}

Fréquemment commenté depuis lors, le lécythe 1129 du Musée National d'Athènes, attribué au Peintre de la Mégère, a été initialement publié par M. Mayer dans un article ancien, intitulé "Noch einmal Lamia»1. L'étude était postérieure à celle que l'auteur avait déjà consacrée au monstre, analysant dans l'Archäologische Zeitung de 1885 une œnochoé du Musée de Berlin, œuvre du Peintre d'Athéna ${ }^{2}$. Précisons que le vase d'Athènes s'insère dans un groupe de lécythes dont la fabrication ne saurait être antérieure aux années $470^{3}$.

La composition portée sur ce monument donne à voir une scène aussi violente qu'insolite : les tortures qu'un groupe de satyres fait subir à une femme nue, liée au tronc d'un palmier. En se fondant à la fois sur la présence de cet arbre méditerranéen, qu'il regardait comme un indicateur d'espace (connotant ici la Libye), et sur les témoignages littéraires (fragment d'Euripide 922 et nombreuses scholies), le savant avait conclu à l'identité de Lamia, rapprochant ainsi l'image des représentations de la comédie satyrique ${ }^{4}$. Cependant, on constate que, dans les articles successifs $(1885,1891)$, il reconnaît Lamia dans deux figures radicalement dissemblables (Pl. I, a; II, a). La «sphinx gorgonéenne» que semble provoquer la satyresse griffue, sur l'œnochoé, n'offre aucune similitude avec la femme taxée de négroïde - représentée sur le lécythe du Peintre de la Mégère. D'un atelier à un autre, Lamia ne saurait être, dans le même temps, indifférem-

1 Lécythe du Musée National d'Athènes, inv. 1129; $A B L$, p. 170-191; p. 266, 1 et pl. 4950, 2; Collignon Couve, 961; AM, 16 (1891), p. 273-312 et pl. 9; AZ (1885), p. 120 et pl. 7, 2; Ausonia (1907), p. 141-185; AJA, 24 (1920), p. 18; AM, 52 (1927), p. 230-234, fig. 17; J.H. BEARDSLEY, The Negro in Greek and Roman Civilisation, 1967, n 111, p. 59; J. BOARDMAN, Athenian Black Figure Vases, 1980, fig. 277; W. RAEK, Barbarenbild, 1981, p. 80 et n. 251; F.M. SNowDEN Jr, Blacks in Antiquity, 1970, p. 155, n. 89 et p. 160, n. 22-23, fig. 89; L'image du noir dans l'art occidental, I, 1976, p. 133.

2 Enochoé de Berlin, inv. $1934: A Z$ (1885), pl. 7, 2. P. d'Athéna. Cf. E.T. VERmeuLe, in Festchrift F. Brommer (1977), pl. 80,3. Une sphinx, au faciès gorgonéen, polymaste, surgit d'une grotte et fait face à une satyresse griffue et velue.

3 C.H.E. HASPELS, $A B L$, p. 170-191; Appendix XXVII, Catalogue p. 266. W. RAEK (supra, n. 1), qui reproduit le document dans son ouvrage, renonce à identifier le personnage féminin.

4 M. MAYER, loc. cit., in $A M, 16$ (1891), p. 273. 
ment reproduite sous les traits d'une femme obèse (lécythe) et sous ceux d'une hybride de gorgone (œnochoé). L'hybride, loin d'être isolée, a d'ailleurs suscité plusieurs peintures, œuvres d'artistes du temps ( $P$. d'Athéna, $P$. de Thésée). Le plus souvent capturé par Héraclès (l'image de l'œnochoé de Berlin fait exception), le monstre est traîné au bout d'une chaîne, à la manière de Cerbère. L'étrange composition a fait l'objet d'études assez récentes, dans lesquelles on a tenté d'identifier cette proie, demeurée néanmoins mystérieuse ${ }^{5}$ : parodie de l'enchaînement de Cerbère, mais accompli par un Héraclès solitaire ? Capture de Lamia ? Les auteurs se sont interrogés sans se prononcer. Il est évident que l'identification proposée par Mayer, en 1885 (Lamia, pour la sphinx de l'œnochoé de Berlin), a pu conditionner cette seconde exégèse. On voit Lamia dans le monstre enchaîné, parce qu'il rappelle morphologiquement la sphinx macromaste que cet auteur avait ainsi dénommée. Ailleurs, mais à partir d'une autre image, on a cru distinguer encore une Lamia-Karkô ${ }^{6}$ (Pl. II, b). L'ogresse velue qui, sur le skyphos béotien, poursuit des jeunes gens fortement caricaturés, semble plus proche, en fait, de la satyresse qui affronte l'hybride de l'œnochoé de Berlin (Pl. II, a) ! Force est de constater que, du moins aux yeux des observateurs modernes, Lamia se révèle sous des aspects aussi différenciés que celui de la créature torturée (lécythe d'Athènes), de la sphinx hybride (groupe de vases, voir la note 5) et de la femelle simiesque (skyphos du Cabirion). Prenant en compte les diverses interprétations, on se trouve dans la situation inconfortable de disposer de trois schémas formels pour couvrir une unique personnalité mythologique... Le monstre ne connaîtrait-il pas de structuration, sinon canonique, du moins relativement fixe ? On pourrait arguer, pour se dérober à la difficulté, que Lamia, qui ressortit au même groupe tératologique qu'Empuse, peut bien être tout cela à la fois, forte du pouvoir de métamorphose qui caractérise sa compagne ${ }^{7}$. Les textes pourtant ne disent

5 E.T. VeRmeULe, Herakles bringt a Tribute, in Festchrift F. Brommer, 1977, p. 297, pl. 80-81; M. REHO-BumbaLOVA, in BABesch, 58 (1983), p. 53-60 et pl. 1-8. Principales images : œnoché de Boston, inv. 98.925; œnochoé de Berlin, inv. 1934; œnochoé de Copenhague Musée National, inv. 834; Fragment de l'Acropole, inv. 1306 (GraefLANGLOTZ, pl. 75); skyphos de Monopoli, Collection particulière.

6 Pl. II, b. Skyphos béotien : JHS, 84 (1964), pl. 5. Sur Lamia-Karkô, voir RE, s.v. Karkô, col. 1954-1955; M. DelCoURT, Héphaïstos ou la légende du magicien, 1982, p. 182; MAC DeRMoTT, op. cit., $\mathrm{n}^{\circ} 324$ : voir la note 11.

7 Gren., 288 sq. : «Voilà que j'aperçois un grand monstre... effrayant. Il prend toutes les formes, tantôt bœuf, tantôt mulet, puis femme ravissante...".

- «C'est un chien à présent».

- «C'est donc Empuse"... 
rien de sa nature protéiforme et les imagiers savent, depuis le haut archaïsme, représenter au besoin les mutations successives ${ }^{8}$.

En dépit des contradictions relevées entre les deux articles, l'hypothèse de lecture qu'a suggérée Mayer pour le lécythe demeure séduisante. Reconnaître Lamia, livrée à la violence des satyres, impose parallèlement de considérer l'hybride (œnochoé de Berlin) comme un monstre secondaire, ou non identifié.

Il paraît possible, en observant la peinture du Peintre de la Mégère, de conforter l'interprétation qui ne s'était pas, depuis lors, définitivement imposée. En effet, d'autres hypothèses avaient été échafaudées par la suite, dont on rappellera brièvement la teneur :

1. Le drômenon. Pour E. Romagnoli (Ausonia, 1907), la séquence figurée sur le lécythe reproduirait un drômenon, c'est-à-dire un drame rituel, construit à partir d'un thème populaire (la femme torturée) et joué dans un sanctuaire.

Il est malaisé de retrouver, sur l'exemplaire, un jeu scénique comparable à celui qui se donnait dans le sanctuaire des Cabires. Les vases découverts dans le Cabirion de Thèbes fournissent un nombre suffisant d'images pour montrer qu'elles étaient généralement traitées sur le mode parodique. Contrairement aux conventions qui régissent cette iconographie de référence, les satyres torturant ne connaissent ni outrances, ni déformations ${ }^{9}$. Seule la victime a été peinte de manière grotesque. L'incohérence, qui tient au télescopage des modes de représentation (dessin conventionnel pour les satyres, charge pour la femme), devrait orienter vers d'autres repères.

2. L'île des Singes. E. Buschor ( $A M, 52$ [1927]), pour sa part, avait vu dans la scène l'illustration d'un épisode étrange - connu du seul Pausanias -, localisé dans l'île des Satyrides ${ }^{10}$. Ayant accosté dans un archipel peuplé

8 Iconographie de Thétis et Pélée (F. BRommer, Vasenlisten ${ }^{3}, 1973$, p. 321-329) ou de l'Hàlios Gérôn (G. AHLBERG-CoRNELL, Herakles and the Sea-Monster in Attic Blackfigure Vase Painting, 1984).

9 Exemples : vases du Cabirion, K. BraUn-TH.E. HAERVERnICK, Bemalte Keramik und Glas aus dem Kabirenheiligtum bei Theben, IV, 1981, pl. 1-8.

10 PAUS., I, 23, 6-7 : «Euphémos, un Carien, disait qu'en naviguant vers l'Italie, il avait été... entraîné vers la mer extérieure. Il racontait qu'il y avait beaucoup d'îles... certaines peuplées d'hommes à l'état sauvage... Les marins appelaient ces îles Satyrides : leurs habitants étaient basanés et ils avaient sur les reins une queue à peine plus petite que celle des chevaux. Ces hommes, lorsqu'ils les aperçurent, coururent au bateau sans pousser le moindre cri, et tentèrent de s'emparer des femmes qui étaient à bord. Dans leur terreur, les marins finirent par débarquer sur 
de singes, et sur le point d'être attaqués, des marins se seraient enfuis après avoir déposé à terre une femme barbare : "les satyres (c'est-à-dire les habitants du lieu) lui firent subir des violences non seulement selon la nature, mais même sur tout le corps".

E. Buschor établit un rapport entre ce témoignage, qui informe qu'une femme barbare a été livrée à la perversité d'une population simiesque, et la peinture qui orne le lécythe. Certes, dans les deux versions, les Satyrides et les satyres s'acharnent à l'humilier et à la mutiler «sur tout le corps». Mais l'adéquation de l'image et du texte, presque trop parfaite, se heurte à deux séries d'objections, iconographiques et chronologiques. Les satyres, parfaitement caractérisés sur le vase et conformes au type usuel dans le répertoire de l'époque Sévère, n'évoquent en rien des singes. Qu'on se reporte aux vases du Cabirion qui attestent, pour la Béotie, l'usage de schémas iconographiques propres à représenter des gorilles ${ }^{11}$. Par rapport à ces modèles, c'est davantage la victime qui appelle la comparaison avec les singes (Fig. 3) ! Rien d'étrange à cette ressemblance, puisqu'il existait dans l'Antiquité une relation d'équivalence entre la laideur féminine et la guenon. Dans l'Assemblée des femmes, v. 1072, un personnage s'interroge en ces termes sur l'identité de l'être qu'il aperçoit : «Est-ce une guenon barbouillée de céruse ou une vieille revenant des légions infernales ?»12. Les tortionnaires du vase n'ont même pas l'apparence des satyres "laineux", à la pilosité plus simiesque, que l'on découvre, parfois à quatre pattes, dans les représentations du dithyrambe ou du drame satyrique ${ }^{13}$.

La lecture se fonde sur un récit de marin que le périégète prétend tenir d'Euphémos, un navigateur Carien ${ }^{14}$. Comment envisager, quand bien même le parallèle est plaisant, qu'un artisan attique, œuvrant vers 470 av. J.-C., ait pu tirer son inspiration d'une histoire si lointaine dans le temps et

l'île une femme barbare, et les satyres lui firent subir des violences non seulement selon la nature, mais même sur tout le corps».

11 Fragment de bol, Heidelberg, inv. 324. Coll. Inst. Arch. inv. S1555, in W.C. MAC DERMOTT, The Ape in Antiquity, 1938, p. 230, pl. IV,1 (= fig. 3 dans la présente étude). La guenon qui importune des jeunes gens a été identifiée comme Lamia-Karkô (supra, n. 6). Sur les singes, voir MAC DERMOTT, op. cit., p. 59 et p. 77-79. Les satyres sont, chez PLINE, H.N., VIII, 216, une race de singes. On se reportera enfin à J.H. BEARDSLEY, op. cit., III, p. 59.

12 Également: Ois., 441; Paix, 1064-1066.

13 P. des satyres laineux, cratère de Syracuse, CVA Italie 17, pl. 14. Voir aussi les choreutes travestis sur le vase de Pronomos (C. CALAME, Images et Société en Grèce Ancienne, 1987, p. 79-88) et sur un vase du Metropolitan Museum de New York, RICHTER-HALL, 1936, pl. 155.

14 Cela revient à dire que l'épisode est bien tardivement relaté, au IIe siècle de notre ère. 
dans l'espace ? Ce témoignage, oral, qui trouve un écho dans les narrations de Hannon et de Ptolémée, ressortit à un genre littéraire très éloigné du théâtre ou de la fable mythologique, sources dominantes du répertoire des imagiers. Il y a de fortes chances pour que les satyres du thiase que le Peintre de la Mégère a reproduits le plus conventionnellement du monde n'offrent aucun lien, ni morphologique, ni parental, avec les Saturidai, habitants de ces îles situées quelque part dans la mer extérieure.

3. La mégère. C.H. Haspels, dans la monographie qu'elle a consacrée aux lécythes à figures noires, a fait de la protagoniste de la scène de torture l'éponyme du peintre (Beldam Painter). L'image montrerait le châtiment d'une "mégère» (qu'elle compare à une Athénienne caricaturée sur une amphore de Bruxelles), punie par ses serviteurs trop longtemps exploités ${ }^{15}$.

Certains détails, qui n'apparaissent que lorsqu'on étudie minutieusement le vase, ont été, dans tous les cas, négligés. La composition, on ne l'a pas dit, met en scène un supplice usuel dans l'Antiquité, qui portait vraisemblablement le nom d'apotympanismos. Il consistait à exposer à un pal, en position debout (images d'Andromède) ou accroupie (images de Prométhée), un condamné à mort ${ }^{16}$. Le motif de l'exposition "assise», confirmé par un passage de Platon, est déjà connu de l'iconographie du haut archaïsme ${ }^{17}$. Certainement usité dans l'imagerie, le schéma de l'apotympanismos suscite d'étonnantes contaminations. Par exemple, le P. d'Edimbourg, illustrant

15 Amphore de Bruxelles, inv. R 279; C.H. HASPELs, $A B L$, p. 20. L'usage du pilon lui suggère cette explication.

16 Platon, Lois, IX, 855c indique la position «assise» du supplicié. L'iconographie confirme l'attitude : les imagiers placent parfois un thrênus, sur lequel Prométhée prend appui (gemmes, relief d'Olympie). ARISTOTe, Const. Ath., 45, 1. On consultera, sur l'apotympanismos en général, E.C.E. OWEN, in JThS, 30 (1928), p. 259-266; A.D. KANEllopoulos, 1923 (Bibl. tès én Athènais Arkaiologikès Hétaireais, 22).

17 Une coupe phénicienne, conservée au Musée de la Villa Giulia, offre peut-être le prototype de la posture assise (M. MORETTI, The National Museum of Villa Giulia, 1963, p. 275, fig. 183). Pour la Grèce, des représentations caractéristiques du supplice de Prométhée sont :

- fragment de bronze du sanctuaire du Ptoion : $B C H, 16$ (1892), pl. X, VIe s.

- plaque d'Olympie, B 4292 (Chronika, $A D, 17$ [1961-62], pl. 137 a).

- ivoire du sanctuaire d'Artémis Orthia (The Sanctuary of Artemis Orthia at Sparta, 1929 [JHS Suppl. 5], pl. C1).

- coupe du Vatican, inv. 16592; P. d'Arkésilas, C.M. STIBBE, Lakonische Vasenmaler, 1972, pl. 63, 1.

- coupe du Peintre d'Haimon, Coll. privée, $A K$, Beih. 1970, 18, 1.

Le fait que, sur la coupe laconienne, Prométhée soit représenté déféquant indique que la scène relève de la farce. 
l'épisode des sirènes, au lieu de représenter Ulysse attaché au mât du navire, a ligoté le héros à une colonne, comme un condamné ordinaire ${ }^{18}$. Au théâtre, dès l'époque où le lécythe a été décoré, le châtiment était montré sur la scène ${ }^{19}$. Plus tard, le supplice réapparaît dans les pièces que Sophocle, puis Euripide, consacrent à Andromède ${ }^{20}$. Plus intéressant pour notre propos, il donne lieu, dans les Thesmophories, à la parodie de l'exposition d'Andromède. Aristophane n'a pas hésité à tirer un parti comique d'un châtiment si terrifiant ${ }^{21}$. Le Peintre de la Mégère peut, en définitive, s'être tourné vers des sources multiples : modèle iconographique antérieur, mises en scènes de théâtre, souvenirs visuels de supplices réels.

L'exposition infamante pouvait, dans la réalité, s'accompagner de sévices complémentaires, allant jusqu'à la mise à mort par transfixion ${ }^{22}$ ou par fracassement ${ }^{23}$. De fait, ici, la femme ligotée subit simultanément la flagellation, le glôssôkoptein (arrachement de la langue ${ }^{24}$ ) ainsi que des tourments sexuels (brûlures), exécutés à l'aide d'une torche. La crémation, non du sexe mais de la toison pubienne, s'apparente peut-être à l'épilation (pratiquée avec de la cendre chaude) qui frappait les adultères ${ }^{25}$. Sur la gauche de l'image, un satyre s'apprête à assommer encore la victime avec un énorme pilon. Le fracassement du crâne, destiné à achever le supplicié, est

18 P. d'Edimbourg, lécythe d'Athènes, inv. 1130 (CC 998); H. KenNER, Das Theater und Realismus, 1954, fig. 20, p. 127; O. TOUCHEFEU, Thèmes Odysséens, 1968, pl. XXIV, $\mathrm{n}^{\circ} 248, \mathrm{p} .148$ et $A B L, \mathrm{n}^{\circ} 27$.

19 Le Prométhée enchaîné date des années 479-467 environ.

20 SOPHOCLE, fr. 135, 450 c; les vases se rapportant à l'Andromède : cratère de Géla, Gr. de Polygnotos, inv. 18, 18, JHS, 84 (1964), fig. 16; péliké de Boston 63.2663, P. des Niobides, F.M. SNOWDEN, Blacks in Antiquity, p. 231, fig. 90; cratère d'Agrigente, P. de la Phiale, $A R V, 1017,53$; hydrie du Musée Britannique $\mathrm{E} 169$, Gr. Ind., $A R V$, 1062. Les peintures suivantes peuvent illustrer l'Andromède d'Euripide : cratère fragmentaire d'Heidelberg 26/69, J.M. MORET, L'Ilioupersis dans la céramique italiote, 1975, pl. 6; amphore de Bari, Musée National de Naples, inv. MH 3225, K. Schauenburg, Perseus, pl. 24, 2; péliké du Musée National de Naples, inv. SA 708, Schauenburg, op. cit., pl. 23, 1; coupe de Tarente, inv. 8928, SchauenBurg, op. cit., pl. 25,1 ; ciste gravée de Préneste, $D S$, s.v. crux, $\mathrm{I}^{2}$, col. 1574.

21 Thesm., 1002-1135.

$22 B C H$, suppl. I (1973), p. 173-181.

23 Cf. note 26.

24 L'ablation de la langue est un châtiment mythique (Philomèle, Lara, par exemple). Voir Ovide, Métam., VI, 412-670; Fastes II, 83. Mais cette mutilation fut aussi en usage dans la réalité : E. PATlagean, Du châtiment dans la Cité, 1984 (Coll. EFRR, 79), p. 409.

25 L'épilation est évoquée par Aristophane, Nuées, 1085. 
- on l'a signalé - historiquement attesté 26 . Sur un cratère du Groupe de Polygnotos, suggéré probablement par le drame sophocléen, l'imagier a placé, à côté d'Andromède, un esclave éthiopien qui frappe à l'aide d'un instrument contondant. Le geste du jeune noir doit être distingué de l'acte, meurtrier, du satyre qui opère sur le lécythe ${ }^{27}$. Le peintre a maladroitement télescopé, du moins doit-on le supposer, deux instants de la scène : l'implantation du poteau et l'exposition elle-même. La fantaisie débridée du Peintre de la Mégère (on serait tenté de parler aujourd'hui de phantasmes) s'appuie, en fait, sur la connaissance de la pénalité en vigueur à l'époque. Qu'elle vienne de l'imagerie antérieure ou qu'elle subisse l'influence des représentations théâtrales, la fiction picturale s'alimente à des données historiques. Loin d'affabuler, le peintre reproduit diverses tortures qui devaient, dans l'esprit des consommateurs antiques (les usagers du vase), évoquer des châtiments très précis. On ne doit pas perdre de vue le contexte, quasiment juridique, dans lequel s'inscrit l'invention graphique. Certes, ainsi qu'on l'a soutenu, le palmier, dont le tronc tient lieu de pal, pourrait fournir quelque indication topographique. Cependant, aussi bien que la Libye (M. Mayer), l'arbre peut connoter les sanctuaires apolliniens, l'île de Délos ${ }^{28}$; en sorte qu'il serait hasardeux de fonder la moindre hypothèse géographique sur ce seul élément. Peut-être la rectitude du tronc a-t-elle, à elle seule, motivé le choix de cet arbre qui, finalement, avec les rameaux de lierre qui zèbrent le champ, indique que la scène se déroule à l'extérieur. Cette précision rattache encore la composition picturale aux supplices officiels qui se pratiquaient, non seulement dehors, mais hors les villes ou aux confins du pays ${ }^{29}$.

Si les tourments représentés correspondent effectivement à des peines réelles, certains accessoires introduits par le peintre proviennent de l'univers de la comédie, qui font basculer l'image vers le genre parodique. Le fouet par exemple, avec lequel l'un des satyres lacère le dos de la femme, associé aux châtiments officiels (SOPHOCLE, Ajax, 111), est un instrument fréquemment mentionné dans les pièces d'Aristophane (Thesm. 1126; 1135; Guêpes, 643; Gren., 620; 635; Nuées, 748; Paix, 747; Cav., 65-68). Le mástix

26 Plut., Pér., 28.

27 Pour ce vase, se reporter à la note 20.

28 Par exemple la pyxide de Ferrare montrant une image allégorique de l'île de Délos : PH. BRUNEAU, Deliaca, in $B C H, 109$ (1985), p. 551-558. On rencontre la même disposition des branchages, jaillissant du palmier axial, dans une scène apollinienne : $A B V 372,124 ;$ CVA Leiden 1, pl. 30.

29 "Aux frontières", dit PlaToN, Lois, IX., 854d. Inversement, le supplice de Mélanthios au chant XXII de l'Odyssée qui se caractérise comme une vengeance privée se perpètre à l'intérieur du palais d'Ulysse. 
symbolise l'autorité et frappe ou menace l'esclave. Ustensile féminin, en revanche, le pilon tient lieu d'arme dans certaines scènes d'Ilioupersis. On y voit les Troyennes le manier et le brandir au-dessus de leurs têtes, de la même manière que le satyre situé à gauche sur le lécythe ${ }^{30}$. Mais au théâtre, détourné de cet emploi héroïque et mythique, l'objet domestique devient dérisoire et engendre le rire. Dans la Paix, Polémos cherche le pilon pour écraser les villes de l'Attique, tandis que, dans les Guêpes, 938, l'ustensile va, en compagnie de l'écuelle, de la râpe, du gril et de la marmite, jusqu'à témoigner à un procès ${ }^{31}$ ! Le thème même de l'épilation intime qu'évoque, sur le vase, la crémation, lorsqu'elle est pratiquée par un satyre sur une toison féminine, doit également émaner de l'imaginaire comique : un artisan contemporain du Peintre de la Mégère, le Peintre du Diosphos, n'a pas hésité à l'illustrer sur un exemplaire conservé à Bâle ${ }^{32}$.

A droite de la composition, un satyre, appuyé sur le bâton dans l'attitude topique du spectateur, assiste aux tourments. Il ne regarde pourtant pas le supplice, mais, présentant sa face, s'en abstrait afin d'établir un relais entre le groupe des actants et le spectateur réel (l'utilisateur du vase). Outre la relation qu'elle impose, la frontalité permet également de représenter la charge émotive, les affects se lisant mal sur un visage de profil. C'est la bestialité la plus rude qu'exprime le faciès fermé et impavide qui nous interpelle ici ${ }^{33}$.

On a souvent mis l'accent sur les traits négroïdes de la femme maltraitée ${ }^{34}$. La forte ensellure, le ventre bombé, pourraient effectivement passer pour des caractéristiques ethniques. Cependant, la chevelure du personnage, hirsute, n'est pas crépue (déjà C.H. Haspels s'était inquiétée de

30 P. de Kléophradès, P. de Brygos (coupe du Louvre, inv. G 152; ARV 369, 1). Làdessus, H.G. BuchHolz, Archaeologia Homerica, Kriegswesen, II (1980) XI, Keule, p. E 319. Iconographie d'Orphée également : ARV 541,7; 1112,4; 1123,7; 10; 96; 1652.

31 Cavaliers, 983; Paix, 259; 269-270; 288.

32 CVA, Bâle 1 (1981), pl. 56.10. Sur le thème, M.I. DAVIEs, Merkins and Modes, in Images et Société en Grèce Ancienne, 1987, p. 243-248 et p. 245, n. 18.

33 Un satyre déchaîné et bondissant «assiste» de la même manière au sparagmos de Penthee sur une coupe de Douris, Coll. Borowski, Toronto (J.-P. VERNANT, P. VIDALNAqUET, Mythe et Tragédie, II, illustration de couverture). Pour les questions de facialité, voir Fr. FronstisI-DuCroux, in La Cité des Images, 1984, p. 147-160, fig. 167, 215, 226; C. CALAME, in Images et Société en Grece Ancienne, 1987, p. 79 : "par le regard, le processus d'énonciation devient confrontation directe avec le récepteur, énonciataire de l'image». On trouvera la liste des satyres (et des centaures) représentés de face dans A. GREIFENHAGEN, Eine attische Schwarzfigurige Vasengattung, 1929, p. 70, nº 23 à 47. Consulter encore $A W, 10$ (1984), p. 89-105.

34 W. RAECK, op. cit., p. 187; F.M. SNOWDEN, op. cit., p. 155; G.H. BEARDSLEY, op. cit., p. 59 . 
ce détail ${ }^{35}$ ). L'impression tient aussi au fait que la peinture a été réalisée selon la technique de la figure noire. Transposée dans la technique de la figure rouge, la morphologie de la créature serait perçue d'une autre manière. Les particularités physiognomiques et anatomiques incriminées appartiennent, en fait, aux conventions de la caricature. Exceptionnelle dans la peinture du Ve siècle ${ }^{36}$, la charge caractérise en revanche la production découverte au Cabirion de Thèbes. On la rencontre encore sur les chous, un peu plus tardifs, en Attique ${ }^{37}$. Gros ventres, jambes grêles, mamelles tombantes pour les femmes, sexes démesurés ou ridés pour les hommes, pilosité constituent les recettes (permanentes, au demeurant) propres à ce mode d'expression ${ }^{38}$. Excès et malformations ne visent pourtant pas ici à ridiculiser un personnage mythique, comme le veut la tradition sur les vases du Cabirion (Ulysse, Jason, Cadmos, par exemple) ${ }^{39}$. Ils deviennent une manière d'exprimer un statut autre qu'idéal (laideur, âge), autre qu'humain. Cette finalité explique que les satyres échappent à la distorsion et que Lamia, seule, la subisse ${ }^{40}$. L'observation attentive du faciès permet de noter que la bouche, ouverte et distendue, dessine une sorte de groin (Fig. 1). Cadmos, sur un skyphos du Cabirion, de $440 \mathrm{ca}$, présente un prognathisme similaire (Fig. 5) qui fait d'ailleurs songer à certains masques de comédie. On rencontre encore un profil féminin lippu, assez comparable, sur un autre vase provenant du sanctuaire thébain ${ }^{41}$. La langue de Lamia, étirée par les tenailles, sort entre les mâchoires qui ne portent, au registre supérieur, que deux dents pointues. Des ridules superposées barrent le front, entre les yeux. La "mégère» frappe par ses traits horrifiques : ce n'est pas une femme qu'on

$35 A B L$, p. 170 : «is it certain that the woman is a negress?".

36 Âge ou ethnie accusés dans quelques cas particuliers : Images et Céramique grecque, 1983, p. 153-167; iconographie de Géras ou de Busiris (F. BROMMER, Vasenlisten ${ }^{3}$, p. 34-36; F. BROMMER, in AA [1952], p. 60-73); Aithra, chenue et tassée (par ex. cratère à volutes de Bologne, inv. 268; P. des Niobides, CVA Italie 33, Bologne 5, pl. 99).

37 La caricature d'un noir sur un vase du P. d'Emporion indique que le genre n'est pas négligé par les peintres de lécythes (lécythe de Géla, inv. 41, CVA, Italie LIV, Géla 3 , pl. 23). Il est exploité aussi sur des bols de Gnathia; à témoin, la danse grotesque d'une hétaïre obèse (JHS, 55 [1940], p. 534, fig. 51).

38 M. MAYER, loc. cit., p. 306, établissait un parallele entre la figure du lécythe d'Athènes et les personnages usuels du répertoire béotien.

39 Circé : G.H. Bearsley, op. cit., $\mathrm{n}^{\circ} 112$ à 115; Cadmos : A. FairBanks, Catalogue of Greek and Etruscan Vases, MFA, Boston, 1928, pl. LXIX, $\mathrm{n}^{\circ}$ 562, 563.

40 J.-M. MORET, EEdipe, la sphinx et les Thébains, 1984, p. 143 et n. 12. Faisant allusion à Lamia, J.-M. Moret évoque plus généralement «la laideur paradigmatique qui réalise au travers du visage l'anticanon de la beauté hellénique».

41 K. Braun, Th.E. Haevernick, op. cit., pl. I, no 9; P. Walters, G. Bruns, Das Kabirenheiligtum bei Theben, I, 1940, p. 100 et pl. 27 (fig. 5 dans le texte). 
torture sous nos yeux, mais un monstre. A ces remarques s'en ajoute une autre qui confirme la nature tératologique de la figure. Il semble qu'elle soit pourvue d'un membre ityphallique (Fig. 4). La malchance fait que la région en question est parsemée d'épaufrures, rendant la lecture délicate ( $\mathrm{Pl}$. I, b). Bien qu'en partie évanide; on devine que le sexe a été travaillé dans la même technique que la langue et la partie supérieure du groin : l'organe a été peint au lavis puis cerné par une incision très accentuée. Si le tracé au vernis dilué a en grande partie disparu, les contours incisés restent lisibles. Ils délimitent le phallus érigé que le satyre accroupi s'apprête à brûler alors qu'il saisit de l'autre main un téton ${ }^{42}$. Le monstre est nettement bissexué. Le tortionnaire ne se méprend point qui dirige d'emblée ses mains vers les pôles symboliques de cette sexualité contre nature : le sein et le phallus. L'ablation de la langue et la flagellation font figure d'épiphénomènes, comparées aux sévices majeurs que représentent ces violences sexuelles. L'iconographie parodique de la sphin ${ }^{43}$, de l'Erinys ${ }^{44}$ n'ignore pas la bissexualité et sait en tirer un parti quasiment pornographique. Dans le contexte qui nous retient cependant, la double nature oriente essentiellement vers Lamia. Car cet épouvantail, aux yeux amovibles comme ceux des Graies, qui se repaît des fœetus arrachés aux ventres des mères ${ }^{45}$ et qui fréquente le cortège des nyktipóloi, a été dépeint sous l'aspect d'un monstre androgyne et repoussant. Aristophane, raillant Cratès, fournit ces précisions ${ }^{46}$. Son témoignage, quoique postérieur à la période à laquelle remonte le lécythe, doit être pris en compte. On a le sentiment qu'il fait référence à une veine populaire, assurément traditionnelle, tant les caractéristiques prêtées à Lamia semblent avoir une valeur proverbiale. Le comique mentionne par deux fois les «testicules» du monstre, opérant au second degré une charge dirigée contre Cléon :

Paix, 758 : «le monstre aux crocs pointus... il avait les testicules infectes de la Lamie...»

42 Je présente un croquis réalisé à partir d'une photographie inédite, pl. I, b, exécutée spécialement, d'après mes instructions, au Musée d'Athènes. A cette occasion, qu'il me soit permis d'adresser mes vifs remerciements à Madame l'éphore Olga Alexandri.

43 J.-M. MORET, Edipe, la sphinx et les Thébains, catalogue, n 188 à 192.

44 Chous attique (440 ca), in $A J A, 50$ (1946), p. 195, fig. 18.

45 «La nuit, elle vient dévorer les foetus en éventrant les femmes enceintes» : Comment. anonyme in Comment. in Aristot. Graeca, t. XX, 1892, p. 427, 38-40, cité par M. DETIENNE, Dionysos mis à mort, p. 143.

46 Cratès ( $450 \mathrm{ca}$ ), voir H. KocK, Sch. Arit. 77, in CAF, I, 136 F, fragment 18 et A. LESKY, Geschichte der Griechischen Literatur, 1963, p. 438. 
Guêpes, 1035 : «... il avait les bourses infectes d'une Lamie» 47.

Enfin, au vers 1178 de la même pièce et bien que le passage demeure obscur, Aristophane - ne négligeant pas l'aubaine d'une précision scatologique - fait allusion à la capture de Lamia :

«d'abord comme la Lamie, se voyant prise, lâcha un pet» 48 .

Le narrateur omet de révéler par qui le monstre fut saisi et ainsi terrorisé... Mais le verbe employé - $\dot{\alpha} \lambda i ́ \sigma \kappa o \mu \alpha \imath$ - signifie être saisi et peut avoir le sens d'être pris en flagrant délit, d'être condamné. C'est dire qu'il renvoie au contexte pénal auquel appartiennent aussi les supplices représentés sur le vase. La relation de l'événement chez Aristophane conforte l'hypothèse avancée pour expliquer l'image du lécythe : Lamia capturée et solidement fixée au pal. Déjà E. Vermeule et $M$. Reho-Bumbalova avaient tiré parti de cette citation afin d'identifier Lamia prise dans la sphinx hybride, plusieurs fois reproduite dans l'entourage du Peintre d'Athéna ${ }^{49}$. Quel qu'ait été son aspect dans les représentations, il est assuré qu'une légende relative à la capture de Lamia circulait en Attique. Redisons seulement que la scène de tortures nous paraît aller davantage dans le sens de la terminologie usitée, pour la narrer ensuite, dans la pièce d'Aristophane. Certaines figurines, plus récentes, qui représentent des personnages du théâtre et qui ont été considérées comme des Lamies, aident à restituer cette figure ${ }^{50}$. Lippues, naines ou obèses, ces courotrophes au rictus de sorcière, qui portent dans les bras des enfants à tête fœetale, offrent des analogies avec la créature torturée. Parfois, même, l'une de ces statuettes est phallique ${ }^{51}$. Lamia, on le devine évasivement par l'importance que lui accordent les railleries d'Aristophane, ressortit au répertoire de la comédie grecque, une appartenance que les terres

47 Le texte est quasiment identique dans les deux pièces (Paix, Guêpes). Dans son intégralité : «le monstre aux dents acérées lui-même, ce monstre dont les yeux, comme ceux de la Cynna, lançaient des regards aux lueurs terribles... Il puait comme un phoque, il avait les bourses infectes d'une Lamie et le derrière d'un chameau».

48 La scholie au vers 77 de l'Assemblée des Femmes insiste sur la nature masculine ( $\dot{\alpha} \rho \sigma \varepsilon v ı \kappa \hat{\varsigma})$ du personnage que l'auteur assimile à Lamia, un portefaix du théatre de Cratès. Dans le passage des Guêpes («d'abord comment la Lamie, au moment d'être prise, lâche un pet, et après comment Cardopion et sa mère..."), Aristophane associe Lamia à un ustensile de la comédie, ici anthropomorphe, le couvercle de la huche à pain (voir le rapprochement avec le pilon, p. 52et n. 30).

49 P. d'Athéna, P. de Thésée, $A B L$, p. 141-165; cf. note 5.

50 M. BIEBER, The History of the Greek and Roman Theater, 1961, p. 248, fig. 823-24; Theaterwesen, pl. 108-112.

51 S. BESQUES, Catalogue des figurines en terre cuite du Louvre, I, 1971, p. 36, D 200, pl. 44 (fin Ve siècle); $A K$, Beiheft 2, 1964, pl. 5 et 7. 
cuites de la fin du Ve siècle ne démentent pas. Le laconisme même de Philocléon, dans les Guêpes, dénote la notoriété de l'historiette qu'il mentionne.

C'est cependant grâce au théâtre populaire italien, par le biais des palliatae et des attelanes, qu'il est possible de mesurer l'ampleur du rôle dévolu à la croquemitaine ${ }^{52}$. Familière de ces farces, aux côtés d'Akkô, d'Alphitô ${ }^{53}$, la dévoreuse nocturne jouissait d'une popularité telle qu'elle finit par lasser... à en croire Horace. Le poète crut bon, s'adressant aux auteurs dans l'Art Poétique, 340, de demander qu'on renonçât à montrer le moment où l'on arrachait du ventre de Lamia l'enfant qu'elle avait dévoré :

«qu'il ne retire pas tout vif du ventre d'une Lamie un enfant dont elle s'est repue... nos graves sénateurs rejettent ce drame» 54 .

La mise en scène dut être fréquemment reproduite, pour avoir à ce point capté l'attention de l'auteur. Leitmotiv de la comédie romaine, la fable peut avoir été diffusée et véhiculée par les récits et les images populaires. Ne peut-on, dès lors, envisager qu'un épisode de ce type avait été illustré (470 ca) par un artisan attique? Le monstre capturé, livré aux satyres, subirait des sévices qui préfigurent - ou équivalent - la scène de l'éventration, plus tardivement relatée par Horace ? Le thème provient du folklore : Lamia, avec Empusa, Mormô, Baubô et quelques autres, peuplent les croyances familières des obstétriciennes et des nourrices. Elle incarne les terreurs qui menacent la grossesse et la petite enfance. Sappho déjà l'assimilait aux Striges ${ }^{55}$, Théocrite, ensuite, la rapprochera de Gello ${ }^{56}$. La figure se classe donc parmi les monstres populaires, nés de la superstition, et s'insère logiquement dans le répertoire du Peintre de la Mégère. On a noté combien cet artisan ainsi que ceux qui gravitent autour de lui se plaisent à illustrer sur les lécythes des thématiques secondaires, marginales et insolites ${ }^{57}$. Le sujet répond en outre aux goûts personnels que ce peintre affiche pour les représentations de tortures. Sur un autre lécythe du Musée d'Athènes, l'artisan a imaginé une

52 PLUT., Demetr., 27; MuNck, De Fabul. Attelan., p. 30.

53 J.-P. CÈBE, La caricature et la parodie, 1966, p. 37-44 : les héritières des sorcières du mime dorien.

54 «Neu pransae Lamiae vivum puerum extrahat alvo», Epitre aux Pisons (Guvres Complètes, J.T. DUBocheT, 1845, p. 386). L'anecdote est mentionnée par M. BIEBER, op. cit., p. 248.

55 Sappho, fr. 47 B 4.

56 Scholie à Théocrite, 15,40 .

57 P. d'Athéna et P. de Thésée : capture de la sphinx hybride (supra, n. 5); P. d'Haimon, $P$. de Diosphos : enfant cuisant dans un chaudron, $A B L, 227,38 ; 241,5.6$. 
scène de noyades collectives, tandis qu'il a organisé, sur un autre exemplaire, une frise de chasseurs de têtes brandissant leurs trophées ${ }^{58}$.

Notre image laisse supposer que des anecdotes populaires, qui alimenteront par la suite le théâtre, circulaient dans les ateliers dès le début du Ve siècle, ou plus tôt. On y retrouve la même verve que sur le cratère Dümmler 59 qui met en scène une exposition au pilori ou que sur un skyphos béotien ${ }^{60}$, montrant les sévices (sexuels notamment) qu'endure un artisan, suspendu par un savant système de poulies au plafond d'un atelier de céramistes ! Ce n'est pas le hasard, mais bien l'existence d'un courant truculent, qui veut que l'on rencontre ces scènes de supplices, relativement caricaturales, entre les années 550 et $470 \mathrm{ca}$; pour les voir ensuite totalement disparaître du répertoire, comme si le théâtre comique avait pris le relais des "livres" d'images que constituent les vases ${ }^{61}$. Tel l'«arroseur arrosé» du cinématographe naissant, Lamia tortionnaire et dévoreuse fut dans l'imagerie populaire (avant que dans la comédie) à son tour torturée, voire éventrée vive. C'est à une telle version parodique que l'on doit songer pour la composition du lécythe d'Athènes, agencée autour de l'épisode de Lamia "prise en flagrant délit».

Université des Sciences Humaines

Monique HALM-TISSERANT

de Strasbourg

Institut d'Archéologie Classique

Palais Universitaire

F - 67084 STRASBOURG

58 Léc. du Musée National d'Athènes, inv. 487 (CC 969); $A B L$, p. 171, pl. 51; P. DuCREY, Le traitement des prisonniers de guerre en Grèce antique, 1968, pl. III. Lécythe du Musée Britannique, inv. B658; $A B L$ 269, $\mathrm{n}^{\circ} 67 ; A B V$ 586; J.R. GREEN, Greek Vases in the J.P. Getty Museum, 3, 1986, p. 112, fig. 21.

59 Cratère corinthien "Dümmler", Musée du Louvre, inv. E 632 : très importante bibliographie; voir notamment A. SEEBERG, Astrabica, in SO, 40-41 (1965-1966), p. 55; Schwerin Papers, 1966, p. 711-712, pl. 83, 2-84.

60 Skyphos béotien du Musée National d'Athènes, inv. $442: \mathrm{H}$. BLÜMMER, in AM, 14 (1982), p. 150-159; I. SCHEIBLER, Griechische Töpferkunst, 1983, p. 20, fig. 108; J.-P. Vernant, M. DeTIENNE, Les ruses de l'intelligence, 1974, p. 185.

61 Les compositions postérieures illustrent les grands supplices mythiques d'Ixion, d'Andromède, de Lycurgue, de Tantale, etc. C'est dire qu'elles échappent au climat spécifique des images de la figure noire, aux confins de la pénalité et de la scatologie. 


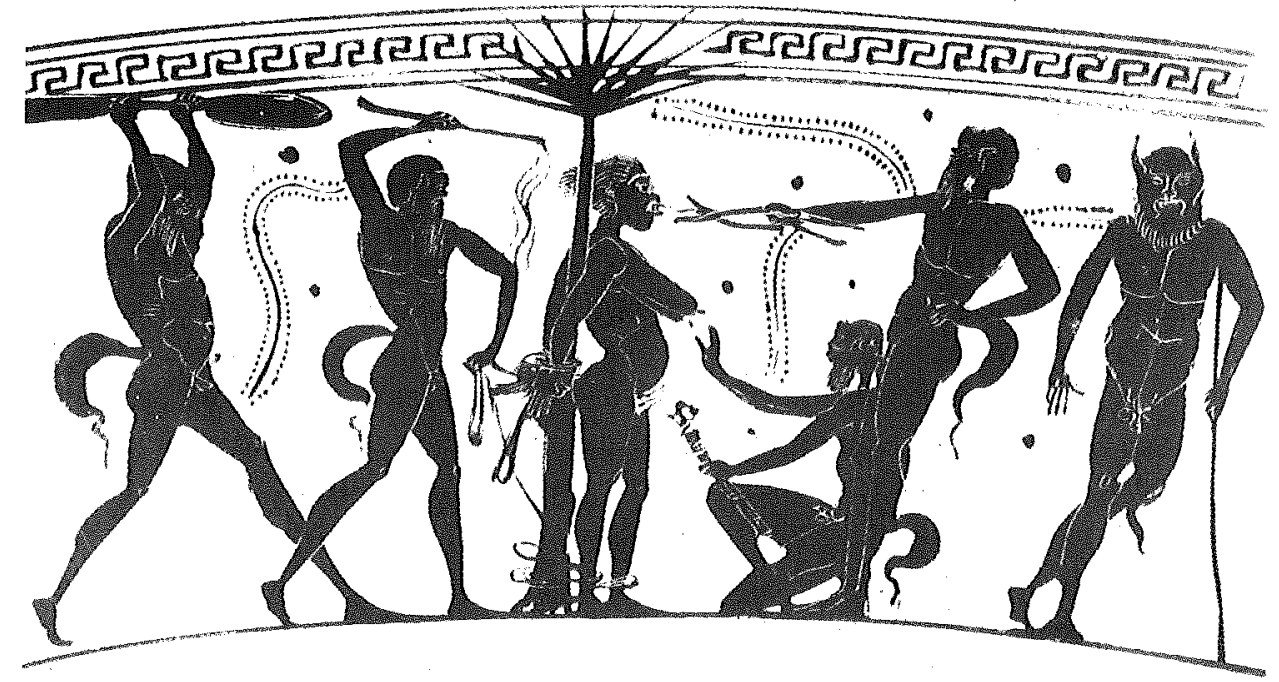

Pl. I, a : Lécythe du Musée National d'Athènes, inv. 1129, d'après F.M. SNowDEN, Blacks in Antiquity, 1970, fig. 89 (ci-dessus).

PI. I, b : Détail du lécythe, inv. 1129 : photo du Musée National d'Athènes (ci-dessous).

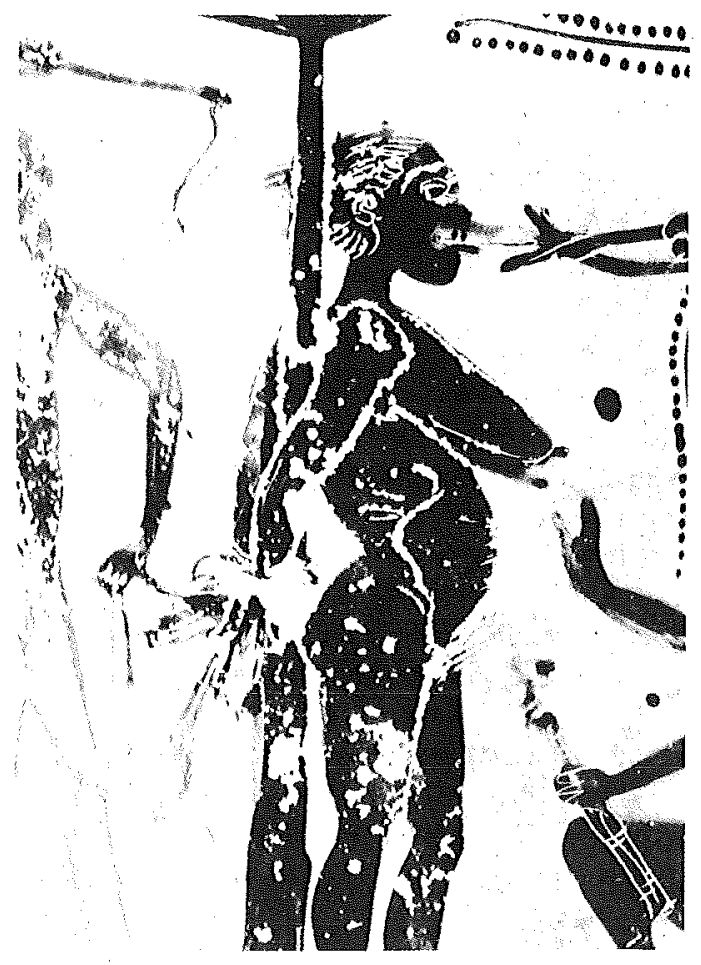




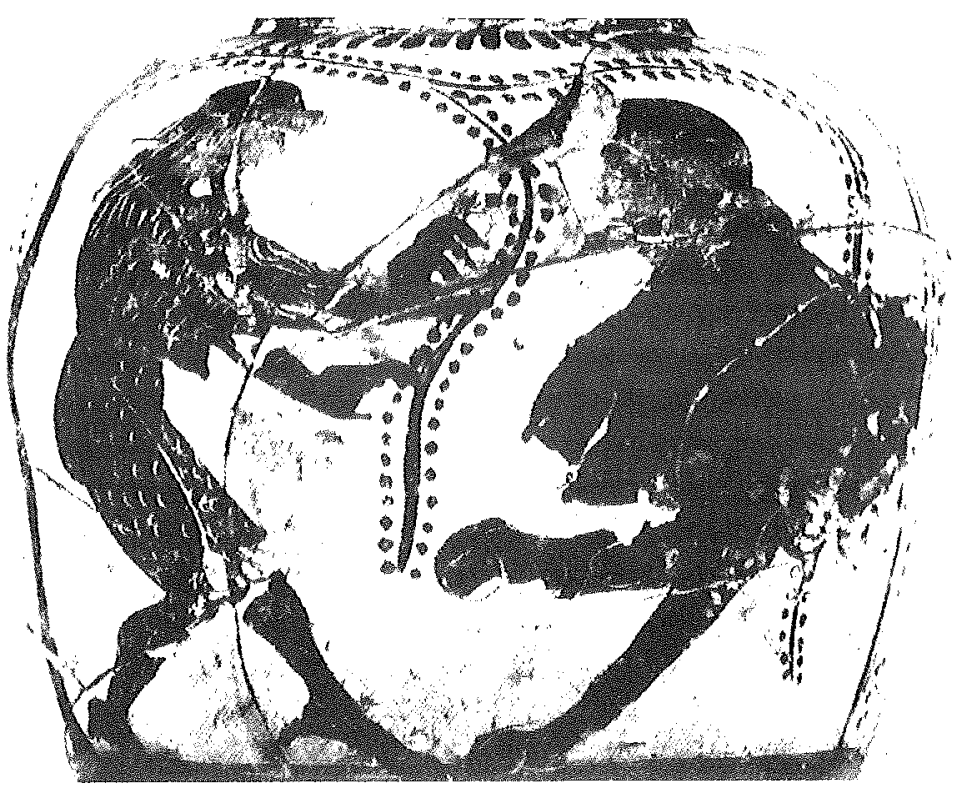

Pl. II, a : Enochoé de Berlin inv. 1934, d'après BABesch, 58 (1983), p. 60, 8 (ci-dessus).

Pl. П, b : Skyphos béotien, Lamia Karkô, d'après JHS, 84 (1964), pl. 5 (ci-dessous).

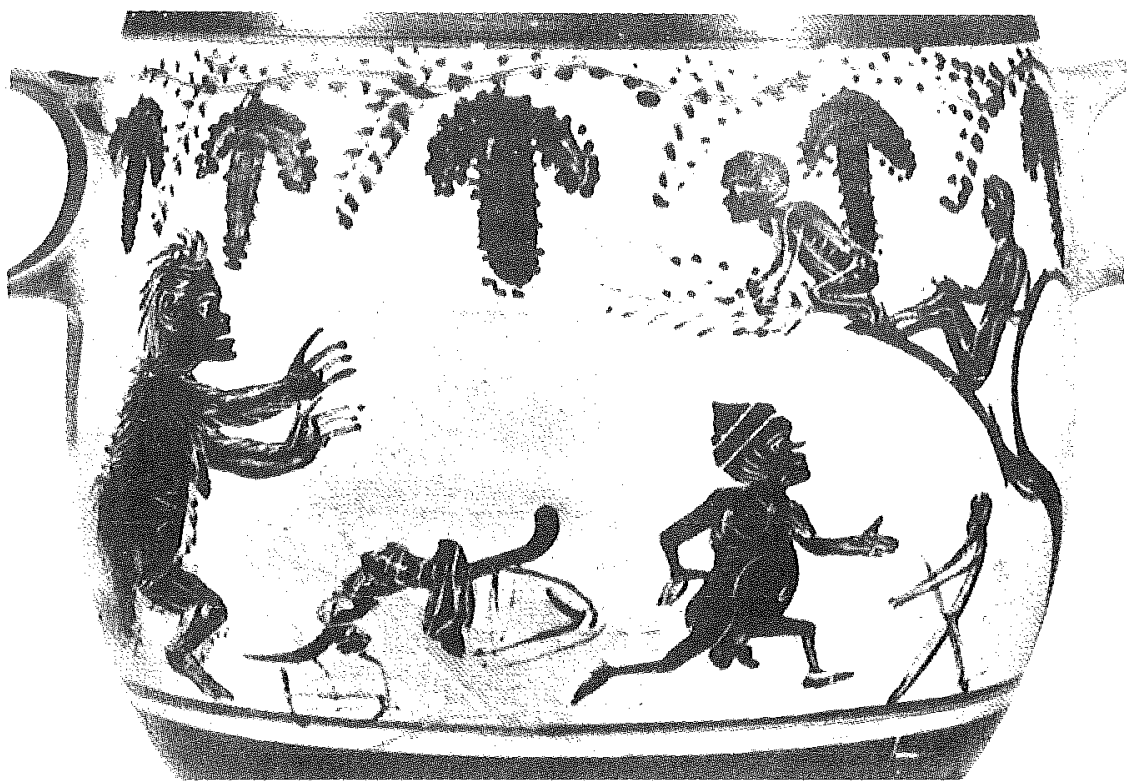




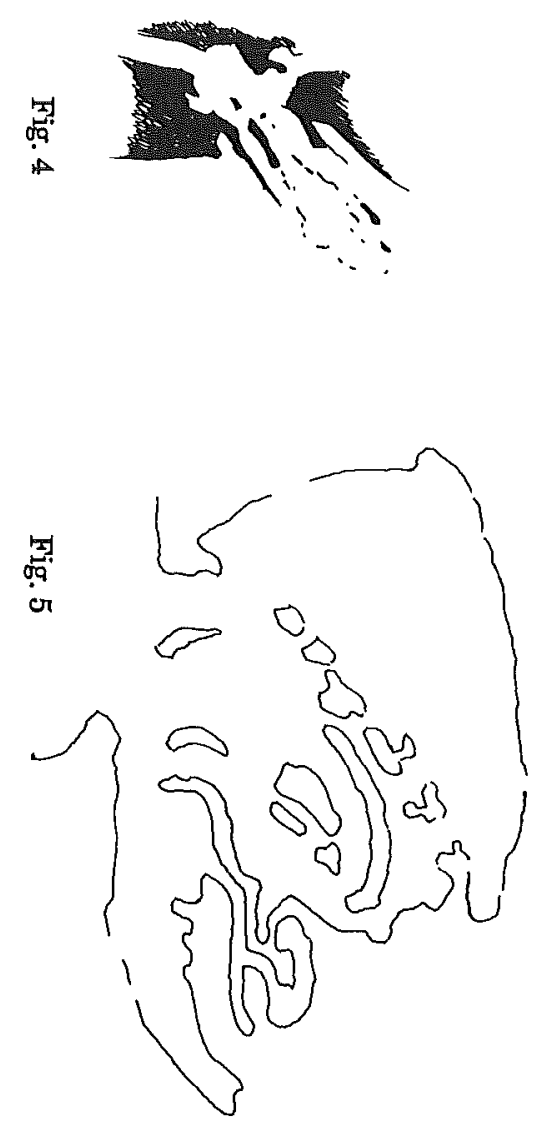

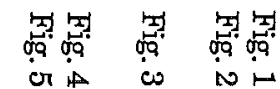

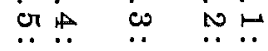
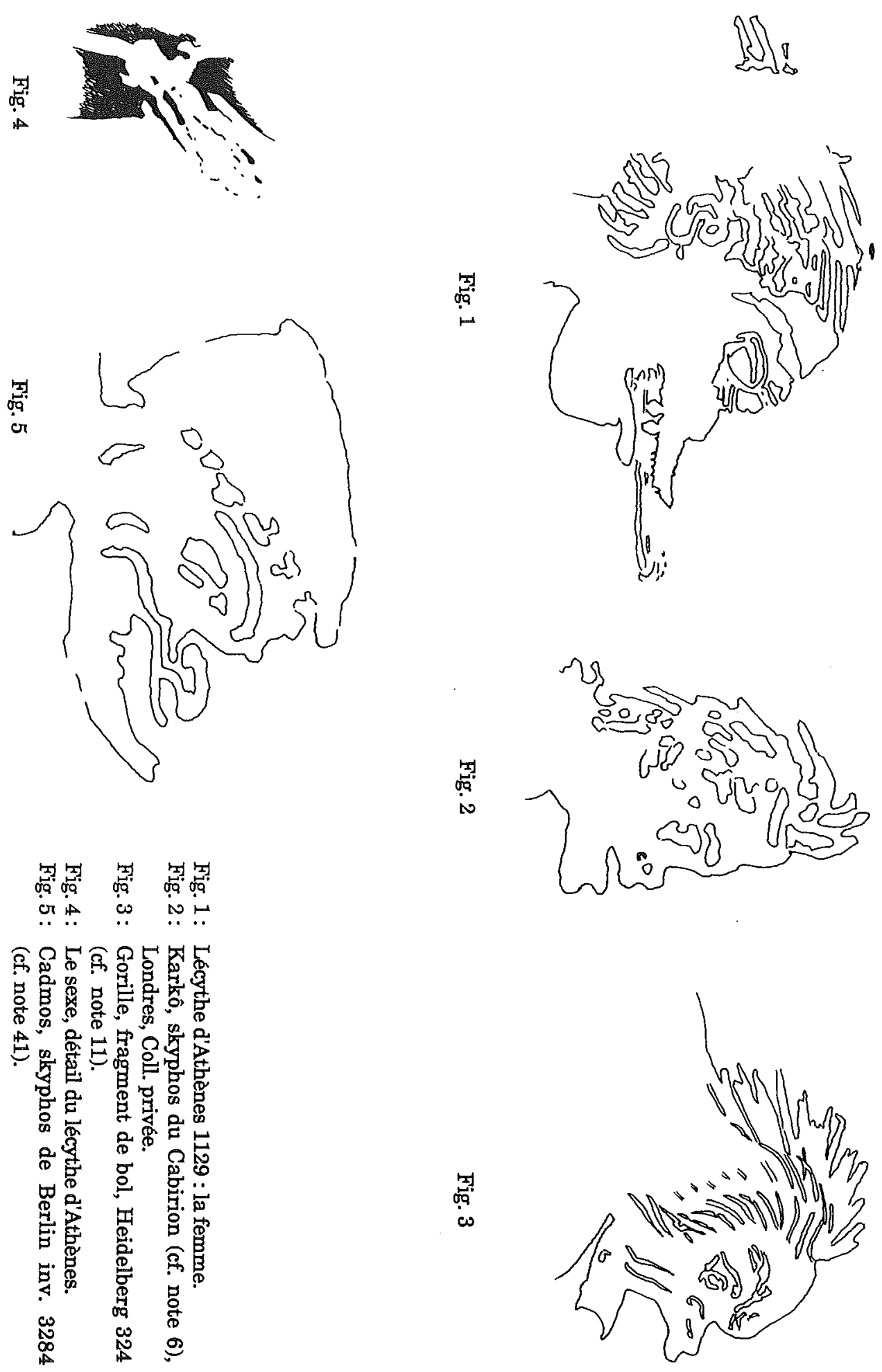